\title{
ACCOUNTS \\ of chemical research
}

\section{Gabapentin: A Stereochemically Constrained $\gamma$ Amino Acid Residue in Hybrid Peptide Design}

\author{
PREMA G. VASUDEV, ${ }^{+}$SUNANDA CHATTERJEE, ${ }^{\ddagger}$ \\ NARAYANASWAMY SHAMALA, ${ }^{+, *}$ AND \\ PADMANABHAN BALARAM ${ }^{\neq, *}$ \\ ${ }^{\dagger}$ Department of Physics, ${ }^{\ddagger}$ Molecular Biophysics Unit, Indian institute of Science, \\ Bangalore 560012, India
}

RECEIVED ON APRIL 6, 2009

\section{CON SPECTUS}

N ature has used the all- $\alpha$-polypeptide backbone of proteins to create a remarkable diversity of folded structures. Sequential patterns of 20 distinct amino acids, which differ only in their side chains, determine the shape and form of proteins. Our understanding of these specific secondary structures is over half a century old and is based primarily on the fundamental elements: the Pauling $\alpha$-helix and $\beta$-sheet.

Researchers can also generate structural diversity through the synthesis of polypeptide chains containing homologated $(\omega)$ amino acid residues, which contain a variable number of backbone
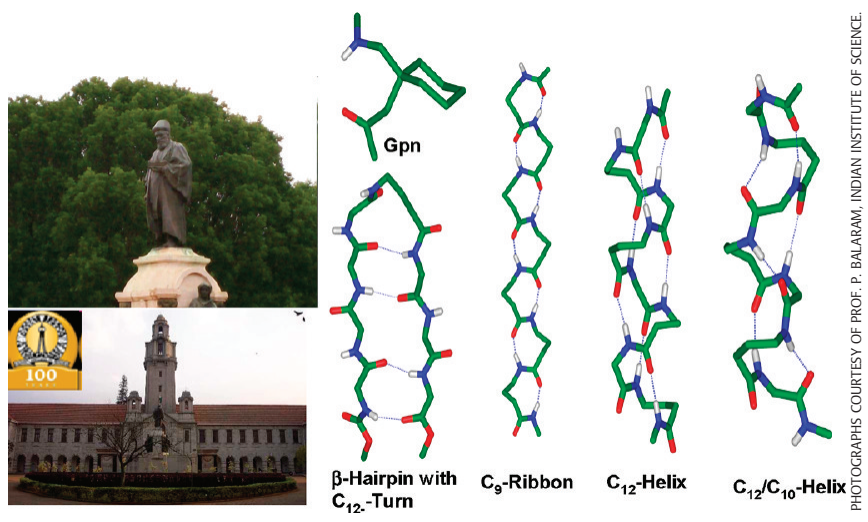
atoms. However, incorporating amino acids with more atoms within the backbone introduces additional torsional freedom into the structure, which can complicate the structural analysis. Fortunately, gabapentin (Gpn), a readily available bulk drug, is an achiral $\beta, \beta$-disubstituted $\gamma$ amino acid residue that contains a cyclohexyl ring at the $\mathrm{C}_{\beta}$ carbon atom, which dramatically limits the range of torsion angles that can be obtained about the flanking $\mathrm{C}-\mathrm{C}$ bonds. Limiting conformational flexibility also has the desirable effect of increasing peptide crystallinity, which permits unambiguous structural characterization by X-ray diffraction methods. This Account describes studies carried out in our laboratory that establish Gpn as a valuable residue in the design of specifically folded hybrid peptide structures.

The insertion of additional atoms into polypeptide backbones facilitates the formation of intramolecular hydrogen bonds whose directionality is opposite to that observed in canonical $\alpha$-peptide helices. If hybrid structures mimic proteins and biologically active peptides, the proteolytic stability conferred by unusual backbones can be a major advantage in the area of medicinal chemistry. We have demonstrated a variety of internally hydrogen-bonded structures in the solid state for Gpn-containing peptides, including the characterization of the $C_{7}$ and $C_{9}$ hydrogen bonds, which can lead to ribbons in homo-oligomeric sequences. In hybrid $\alpha \gamma$ sequences, distinct $C_{12}$ hydrogenbonded turn structures support formation of peptide helices and hairpins in longer sequences. Some peptides that indude the Gpn residue have hydrogen-bond directionality that matches $\alpha$-peptide helices, while others have the opposite directionality.

We expect that expansion of the polypeptide backbone will lead to new classes of foldamer structures, which are thus far unknown to the world of $\alpha$-polypeptides. The diversity of internally hydrogen-bonded structures observed in hybrid sequences containing Gpn shows promise for the rational design of novel peptide structures incorporating hybrid backbones.

\section{Introduction}

The diversity of intramolecularly hydrogenbonded structures in synthetic polypeptides has greatly expanded following the introduction of backbone homologated $\beta$ and $\gamma$ amino acid resi- dues in the area of peptide design. ${ }^{1-9}$ The introduction of additional atoms into peptide chains increases the number of degrees of torsional freedom at each residue. While the conformational properties of $\alpha$ amino acids in peptides and pro- 


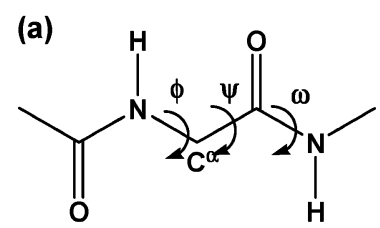

$\alpha$-residue

(b)<smiles>CNC(=O)NC(C)(C)[C@@H](C)NC(C)=O</smiles>

$\alpha$-aminoisobutyric acid

(Aib)

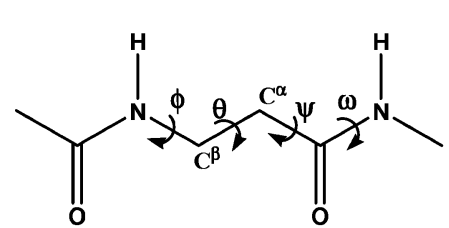

$\beta$-residue

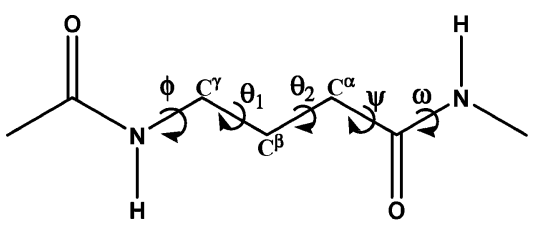

$\gamma$-residue

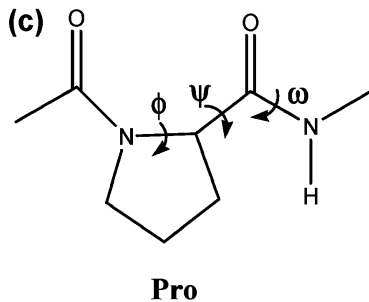

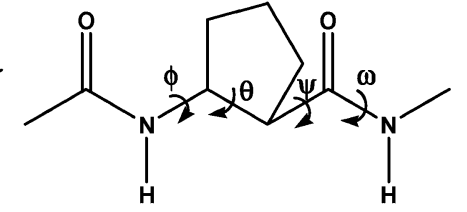

trans 2-aminocyclopentane carboxylic acid (trans-2-ACPC)

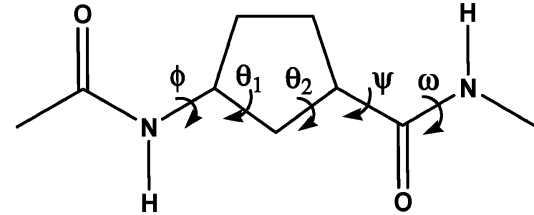

trans 3-aminocyclopentane carboxylic acid (trans-3-ACPC)

FIGURE 1. (a) Definition of backbone torsion angles for $\alpha, \beta$, and $\gamma$ amino acid residues, (b) geminally disubtituted $\alpha$, $\beta$, and $\gamma$ residues, and (c) $\alpha, \beta$ and $\gamma$ residues in which side chain-backbone cyclization restricts the backbone torsional freedom.

teins are conveniently described by the two Ramachandran angles $\phi$ and $\psi$, the analysis of $\beta$ residues includes three variables, $\phi, \theta\left(\mathrm{C}^{\beta}-\mathrm{C}^{\alpha}\right)$, and $\psi$, and for $\gamma$ amino acid residues, four variables, $\phi, \theta_{1}\left(\mathrm{C}^{\gamma}-\mathrm{C}^{\beta}\right), \theta_{2}\left(\mathrm{C}^{\beta}-\mathrm{C}^{\alpha}\right)$, and $\psi$, are required (Figure 1). ${ }^{10}$ The generation of folded peptide backbones in sequences containing higher homologues of the $\alpha$ amino acids requires the population of gauche $\left(g^{+} / g^{-}, \theta \approx \pm 60^{\circ}\right)$ conformations about the additional $\mathrm{C}-\mathrm{C}$ bonds. Current interest in the area of peptides with hybrid backbones stems from the observation of novel hydrogen-bonded structures in $\beta$ peptide homo-oligomers and in hybrid sequences containing $\alpha, \beta$, and $\gamma$ amino acids. ${ }^{6-8}$ The stability of peptides containing $\beta$ and $\gamma$ residues to the action of proteases ${ }^{4}$ provides an additional impetus for systematic studies of hybrid peptide conformations, which may lead to the rational design of analogs of medicinally important peptide sequences., ${ }^{9,1-13}$ Design strategies are greatly aided by the availability of conformationally constrained residues in which the range of accessible backbone structures is limited. Conformational space available to amino acid residues may be restricted by side chain-backbone cyclization or by backbone alkylation (Figure 1). In the case of $\alpha$ amino acid residues, the two pre-eminent examples are the proteinogenic amino acid proline, in which the dihedral angle $\phi\left(\mathrm{N}-\mathrm{C}^{\alpha}\right)$ is limited by pyrrolidine ring formation, and the nonprotein amino acid $\alpha$-aminoisobu- tyric acid (Aib), in which gem dialkyl substitution at $\mathrm{C}^{\alpha}$ results in limiting the range of both $\phi$ and $\psi$ angles. ${ }^{14-18}$ In the case of $\beta$ residues, 2-aminocyclopentane carboxylic acid (2-ACPC) in which $\theta$ is restricted approximately to $85^{\circ}$ has proved remarkably useful in the construction of novel helical structures. $^{3,4}$ In this Account, we present an overview of the use of the achiral $\beta, \beta$-disubstituted $\gamma$ amino acid residue 1 -aminomethylcyclohexaneacetic acid (gabapentin, Gpn) in the design of peptides with diverse intramolecularly hydrogenbonded structures, including peptide ribbons and helices.

Gabapentin (Neurontin) is a widely used antiepileptic drug, which is also currently prescribed for the treatment of neuropathic pain. ${ }^{19}$ The large scale production of the bulk drug makes the amino acid residue readily available for peptide synthetic purposes. Its achiral nature is an added advantage, permitting the handedness of hybrid secondary structures to be determined by the configuration of the $\alpha$ amino acids used. Gabapentin was originally produced in a search for analogs of the neurotransmitter $\gamma$-aminobutyric acid ( $\gamma \mathrm{Abu}, \mathrm{GABA})$. Ironically, gabapentin did not turn out to be a ligand recognized by the GABA receptor but nevertheless has proved clinically and commercially important. ${ }^{19}$ In the area of peptide design, gabapentin may be used as a stereochemically constrained analog of the parent unsubstituted $\gamma$ amino acid residue, $\gamma$ Abu. A noteworthy feature of the Gpn residue is the pres- 
(a)

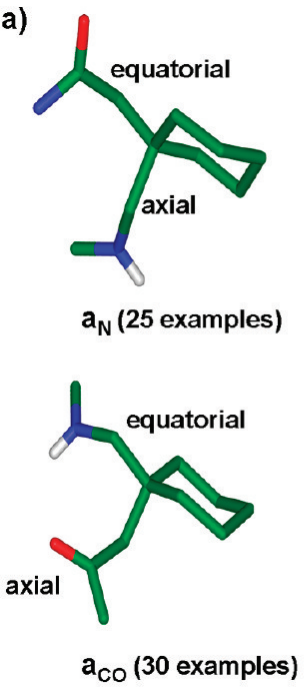

(b)

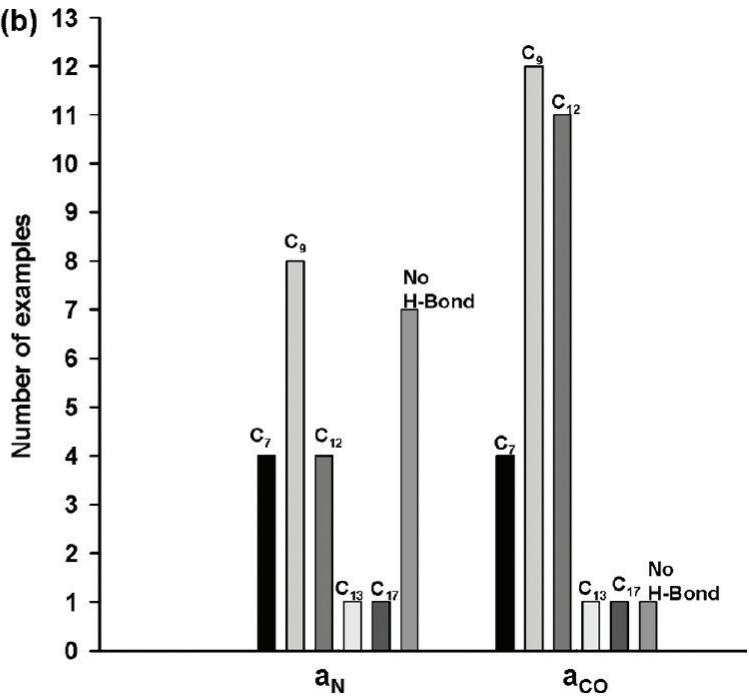

FIGURE 2. (a) Conformers of Gpn, which differ in the orientation of substituents on the cyclohexane ring (aminomethyl group axial, $a_{N}$; carboxymethyl group axial, $\mathrm{a}_{\mathrm{CO}}$ ), and (b) the distribution in crystal structures of $\mathrm{a}_{\mathrm{N}}$ and $\mathrm{a}_{\mathrm{CO}}$ conformers in various internally hydrogen-bonded and non-hydrogen-bonded Gpn residues. Hydrogen bond types are indicated on the top of each bar. Positional disorder, indicating the coexistence of both $\mathrm{a}_{\mathrm{N}}$ and $\mathrm{a}_{\mathrm{CO}}$ conformers, is observed in four examples.
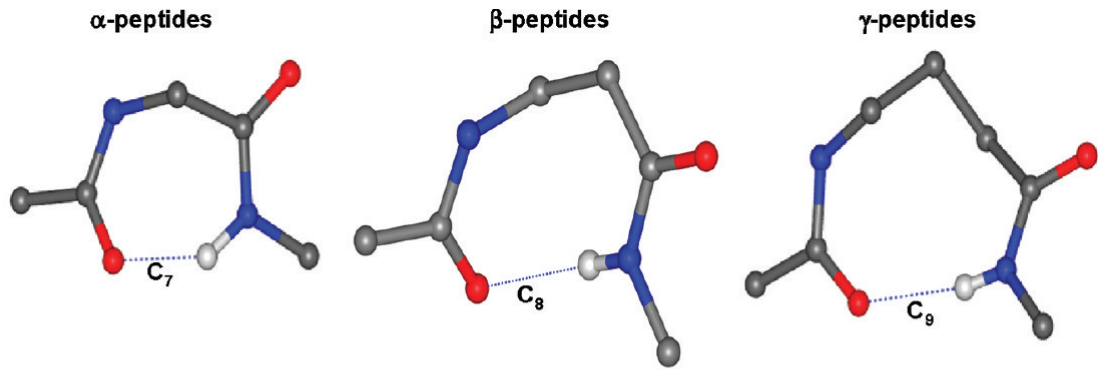

$\mathrm{CO}_{1} \leftarrow \mathrm{NH}_{\mathrm{l}+2}$
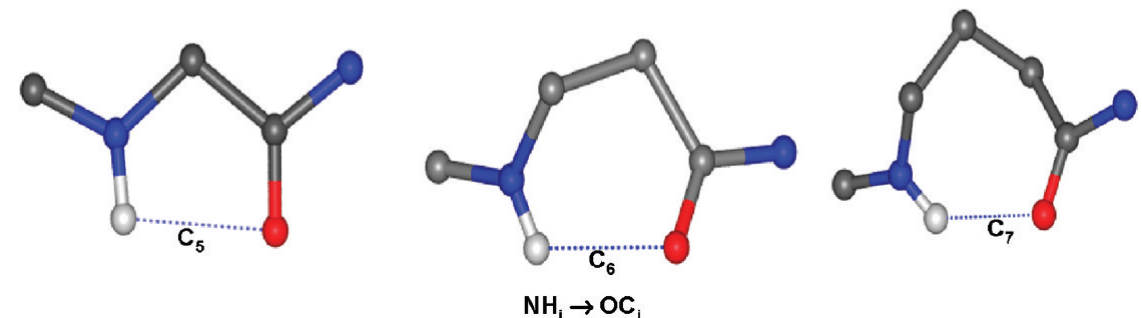

$\mathrm{NH}_{\mathrm{i}} \rightarrow \mathrm{OC}$

FIGURE 3. Hydrogen-bonded turns that are defined by the backbone torsional angles of a single residue in $\alpha$-, $\beta$-, and $\gamma$-peptides.

ence of a 1,1-disubstituted cyclohexane ring, which can exist in two distinct conformations, in which the aminomethyl and carboxymethyl substituents interconvert between axial and equatorial orientations. A large number of crystallographic studies on the amino acid Gpn and several derivatives carried out in this laboratory suggest that the cyclohexane ring conformations do not substantially influence the nature of hydrogen-bonded conformations, which depend solely on the torsional variables at the Gpn residue. Figure 2 provides crystallographically determined examples of the Gpn residue in which the orientations of the substituents at position 1 are interchanged and summarizes the distribution in available Gpn peptide crystal structures. Figure 3 compares the hydrogen- bonded structures that may be generated by involving interactions within a single residue and between backbone amide groups of the flanking residues. For an $\alpha$ residue, two distinct structures have been characterized. These are the $C_{7}$ hydrogen-bonded conformation, also referred to as the $\gamma$-turn, ${ }^{20}$ and $\mathrm{a}_{5} \mathrm{C}_{5}$ conformation, which is observed in the fully extended peptides, most notably in the crystal structures of $\alpha, \alpha$-diethylglycine (Deg) and $\alpha, \alpha$-dipropylglycine (Dpg). ${ }^{21}$ The $\mathrm{C}_{7}$ structures have been observed in proteins and cyclic peptides, but relatively few examples exist in linear peptides. ${ }^{22}$ The directionality of hydrogen bonding in the case of the $C_{7}$ structure involves an acceptor $\mathrm{CO}$ group at the $\mathrm{N}$-terminal end and a donor $\mathrm{NH}$ group at the $\mathrm{C}$-terminal end $\left(\mathrm{CO}_{i} \leftarrow \mathrm{NH}_{i+2}\right)$. In 
(a)

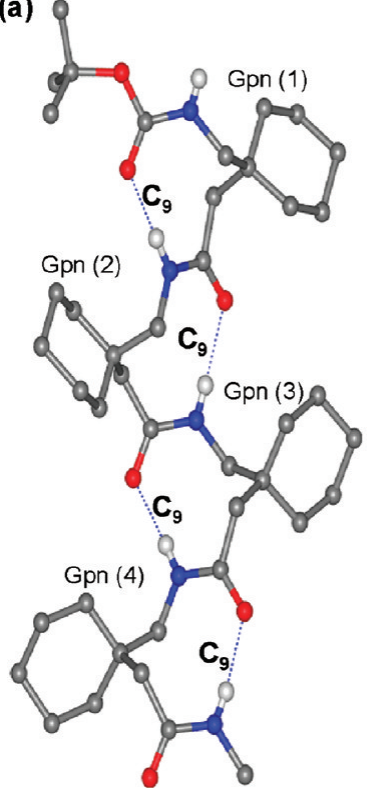

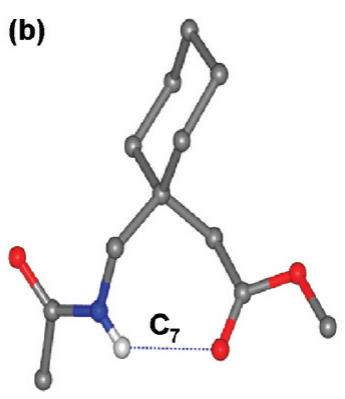

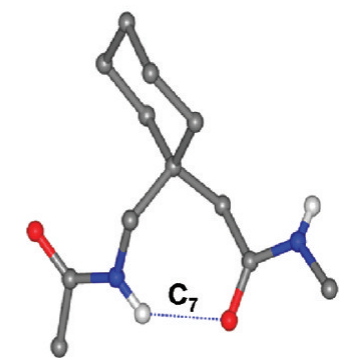

FIGURE 4. (a) $\mathrm{C}_{9}$ ribbon formed by Boc-Gpn-Gpn-Gpn-Gpn-NHMe ${ }^{25}$ and (b) $\mathrm{C}_{7}$ hydrogen-bonded turn in Boc- $\mathrm{AC}_{6} \mathrm{C}-\mathrm{Gpn}-\mathrm{OMe}^{27}$ (top, carboxymethyl group axial) and in Boc-Gpn-Aib-Gpn-Aib-OMe ${ }^{27}$ (residue 3, bottom, aminomethyl group axial).

contrast, the intraresidue $C_{5}$ hydrogen bond has the opposite directionality. In $\beta$ residues, the insertion of an additional carbon atom into the backbone results in an expansion to the corresponding $C_{8}$ and the $C_{6}$ hydrogen bonds. ${ }^{23,24}$ There are relatively few crystallographically characterized examples of these hydrogen bonding patterns in $\beta$ amino acid derivatives. Further expansion to the $\gamma$ amino acid residue yields the analogous hydrogen-bonding patterns, resulting in $C_{9}$ and $C_{7}$ structures. Homo-oligomeric Gpn peptides preferentially adopt $\mathrm{C}_{9}$ ribbon structures in crystals as established from studies on Boc-(Gpn $)_{n}$-NHMe $(n=2,4) .{ }^{25}$ The $C_{7}$ conformation has been observed for Gpn residues at the C-terminus of short peptides and also for internal Gpn residues in larger sequences (Figure 4). ${ }^{26,27}$ When placed adjacent to an $\alpha$ amino acid residue, the $\alpha \gamma$ or $\gamma \alpha$ segments containing Gpn can, in principle, form two residue turns with $C_{12}$ intramolecular hydrogen bonds. ${ }^{28}$ These conformational features would be backbone expanded analogs of the widely studied two residue $\alpha \alpha, C_{10}$ turns (referred to as $\beta$-turns, in the extensive literature of peptides and proteins). ${ }^{29}$

In the case of $\alpha \alpha$ segments, the type I/III $\beta$-turn is a structure in which both residues have approximately the same values for the backbone torsion angles $\phi, \psi\left(-60^{\circ},-30^{\circ}\right)$, permitting repetition of the turn to yield the polypeptide $3{ }_{10}$-helix. ${ }^{17,30}$ In contrast, the type II $\beta$-turn in $\alpha \alpha$ segments has residues at $(i+1)$ and $(i+2)$ in two distinct conformations $\left(\phi_{(i+1)}=-60^{\circ}, \psi_{(i+1)}=120^{\circ}, \phi_{(i+2)}=80^{\circ}, \psi_{(i+2)}=0^{\circ}\right)$, pre-
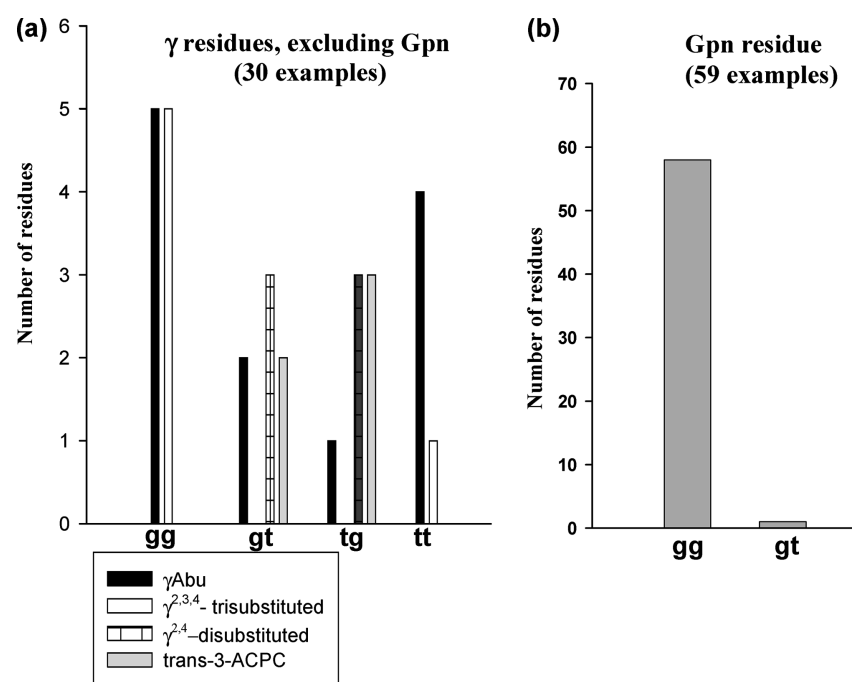

FIGURE 5. Distribution of $\theta$ values in crystal structures of (a) all $\gamma$ residues, excluding Gpn, and (b) Gpn. The average values for $\theta_{1}$ (deg) and $\theta_{2}$ (deg) in panel a are $\theta(\mathrm{gg}), 10$ examples, $\theta_{1}=66.0 \pm$ 5.8, $\max =76.5, \min =58.0 ; \theta_{2}=60.8 \pm 5.9, \max =69.1, \min =$ 50.5; $\theta(g t), 7$ examples, $\theta_{1}=74.6 \pm 22.9, \max =107, \min =50.2$; $\theta_{2}=170.8 \pm 8.1, \max =177.9, \min =159.4 ; \theta(\mathrm{tg}), 7$ examples, $\theta_{1}$ $=162.2 \pm 12.8, \max =175, \min =142 ; \theta_{2}=77.1 \pm 12.0, \max =$ 93.7, $\min =62.3 ; \theta(t t), 5$ examples, $\theta_{1}=174.4 \pm 2.5, \max =$ $176.9, \min =171 ; \theta_{2}=168.7 \pm 18.9, \max =178.6 ; \min =135$. The values for $\theta_{1}$ (deg) and $\theta_{2}$ (deg) averaged over 58 examples for the $g g$ conformation in panel $\mathrm{b}$ are $\theta_{1}=66.0 \pm 5.8, \max =76.5$, $\min =58.0 ; \theta_{2}=60.8 \pm 5.9, \max =69.1, \min =50.5$; and the values for the $g t$ conformation are $\theta_{1}=67, \theta_{2}=-167$. (In the case of centrosymmetric space groups, Gpn residues of only one hand are considered.) (Two examples were not considered in the statistics, because they are close to the eclipsed conformation).

cluding helix formation, with isolated turns facilitating reversal of polypeptide chain direction. ${ }^{14}$ The "mirror image" turns type $\mathrm{I}^{\prime}$ and type $\mathrm{II}^{\prime}$ in which the signs of the torsion angles are inverted, serve as the turning segment in registered $\beta$-hairpin structures in proteins and designed peptides. ${ }^{14,31}$ In hybrid $\alpha \gamma$ sequences, these features of $\alpha \alpha$ segments can be largely retained with the additional atoms incorporated within the hydrogen-bonded loop. In addition, novel hydrogen bond patterns in which the directionality is reversed can be generated.

Ongoing studies in this laboratory focus on delineation of the conformational properties of synthetic peptides containing the Gpn residue. Conformational analysis has been facilitated by the tendency of Gpn-containing peptides to crystallize, permitting X-ray diffraction analysis. Figure 5 compares the distribution of conformation about the $\mathrm{C}^{\gamma}-\mathrm{C}^{\beta}$ and $\mathrm{C}^{\beta}-\mathrm{C}^{\alpha}$ bonds $\left(\theta_{1}\right.$ and $\left.\theta_{2}\right)$ for $\gamma$ amino acid residues in peptide crystal structures with the available results for Gpn residues. In the case of the unsubstituted $\gamma$ amino acid residue $\gamma$ Abu, $\alpha, \beta, \gamma$-trisubstituted, and $\alpha, \gamma$-disubstituted $\gamma$ residues, both gauche $(g)$ and trans $(t)$ conformations about the $\mathrm{C}-\mathrm{C}$ 
bonds are observed. In the case of Gpn residues, the distribution is overwhelmingly in favor of $g g$ conformations. Thus the Gpn residue offers an opportunity for design of hybrid peptide foldamers in which the propensity for the gg conformation can be used to direct structure formation. Interestingly, of the crystallographically characterized $\gamma$ amino acid residues, there are as many as 59 examples for Gpn and only 30 for other $\gamma$ amino acid residues. For the constrained $\gamma$ amino acid residue trans-3-ACPC (Figure 1), six crystallographically characterized examples are available, out of which five adopt tg/gt, while one exhibits an almost eclipsed conformation $(\theta$ $\approx 120^{\circ}$ ).

The use of the Gpn residue in expanding the backbone in conventional polypeptide helices is exemplified by three structures illustrated in Figure 6. Boc-Aib-Gpn-Aib-Gpn-OMe ${ }^{28}$ has two consecutive $C_{12}$ hydrogen-bonded $\alpha \gamma$ turns, which may be formally viewed as backbone expanded analogs of $C_{10}$ turns in a polypeptide $3_{10}$-helix. The structure of the octapeptide Boc-Gpn-Aib-Gpn-Aib-Gpn-Aib-Gpn-Aib-OMe ${ }^{32}$ reveals a regular $\alpha \gamma \mathrm{C}_{12}$ helix over the segment Aib(2) to Aib(6). The $\mathrm{N}$ - and C-terminal Gpn residues adopt the $C_{9}$ hydrogen-bonded conformation. Gpn residues can be accommodated as guests in host $\alpha$ amino acid sequences generating mixed helical structures in which $C_{10}$ hydrogen bonds are formed in the $\alpha \alpha$ segments and $C_{12}$ hydrogen bonds in the $\alpha \gamma / \gamma \alpha$ segments as seen in the structure of $\alpha \gamma \alpha \alpha \gamma \alpha$ sequence Boc-Leu-Gpn-AibLeu-Gpn-Aib-OMe. ${ }^{33}$ These structures permit determination of the torsional parameters necessary for the generation of the regular $\alpha \gamma \mathrm{C}_{12}$ helix (Figure $6 \mathrm{~d}$ ).

The structure determination of the tetrapeptide Boc-LeuGpn-Leu-Aib-OMe, provides an example of an alternative helical conformation that is accessible in the $\alpha \gamma$ sequences (Figure 7a). ${ }^{34}$ In this structure, the Leu(1)-Gpn(2) segment forms a $C_{12}$ hydrogen-bonded turn, which has backbone torsional parameters distinctly different from those observed in the helical $\alpha \gamma$ sequences. Furthermore, the Gpn(2)-Leu(3) segment forms a $\mathrm{C}_{10}$ hydrogen-bonded turn, in which the directionality of the hydrogen bond is reversed. Interestingly, this mixed $C_{12} / C_{10}$ structure can be propagated to yield a novel $\mathrm{C}_{12} / \mathrm{C}_{10}$ helix in a repeating $\alpha \gamma$ sequence (Figure $7 b)$. An important difference between the two types of $\alpha \gamma$ structures is the conformation adopted by the $\alpha$ residue. In the regular $\alpha \gamma \mathrm{C}_{12}$ helix, the $\alpha$ residue adopts a helical $\left(\alpha_{R} / \alpha_{L}\right)$ conformation, whereas in the mixed $C_{12} / C_{10}$ helix, the $\alpha$ residue lies in the semiextended polyproline $\left(\mathrm{P}_{\mathrm{II}}\right)$ region of conformational space.
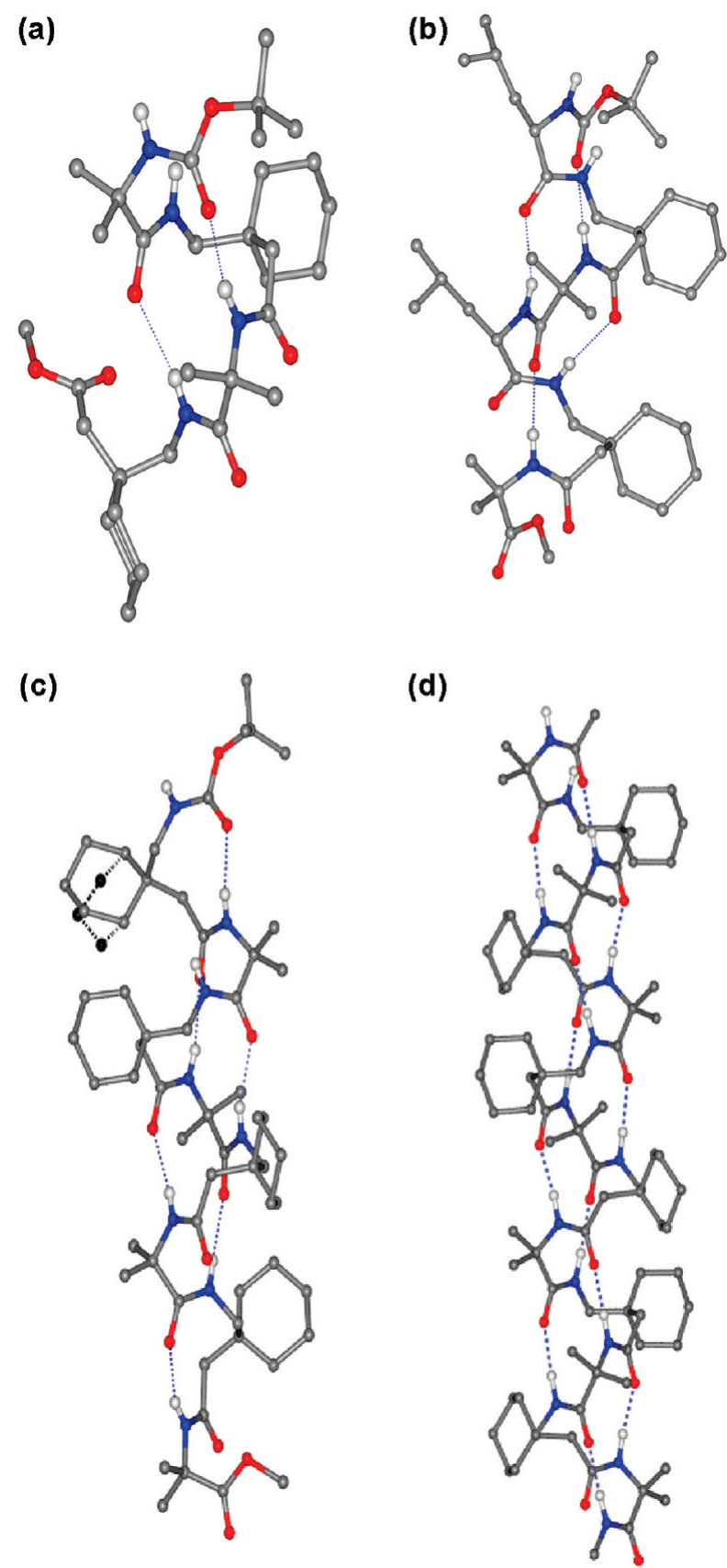

FIGURE 6. Hydrogen-bonded structures in $\alpha \gamma$ hybrid peptides of Gpn: (a) Boc-Aib-Gpn-Aib-Gpn-OMe;28 (b) Boc-Leu-Gpn-Aib-Leu-GpnAib-OMe; $^{33}$ (c) Boc-Gpn-Aib-Gpn-Aib-Gpn-Aib-Gpn-Aib-OMe; ${ }^{32}$ (d) a model $C_{12}$ helix generated using the parameters derived from crystal structures.

\section{Hydrogen-Bonded Turns in Gpn Peptides}

The stereochemical constraints imposed by dialkyl substitution at the $C^{\beta}$ carbon atom in Gpn are compatible with a wide range of intramolecularly hydrogen-bonded structures in hybrid sequences. For $\alpha \gamma$ segments, three distinct kinds of $C_{12}$ hydrogen-bonded structures have been experimentally characterized in crystals (Figure 8). The type I $\alpha \gamma$ turn corresponds to a helical turn and is an expanded analog of 
(a)

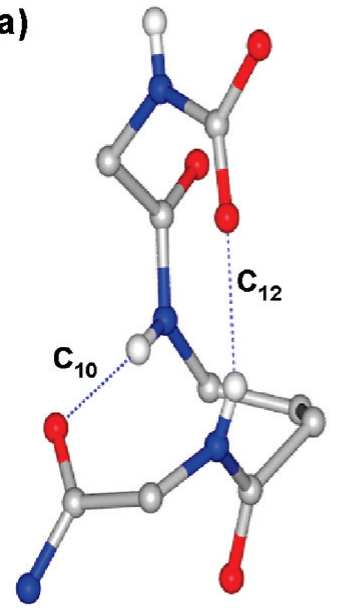

(c)

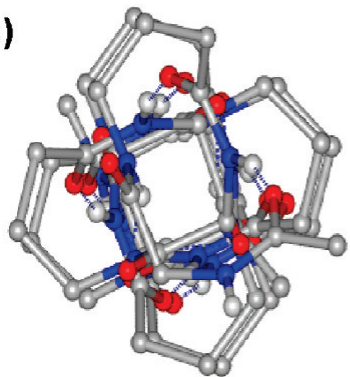

(b)

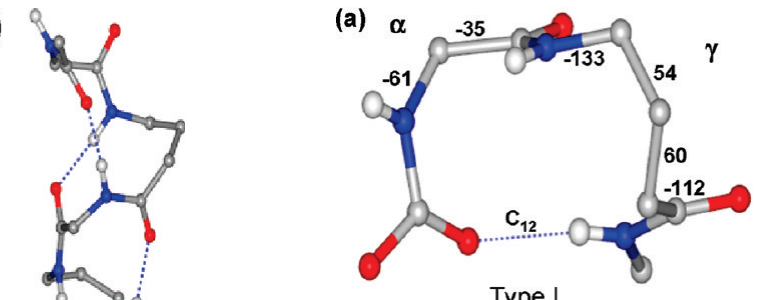

Type I

(b)

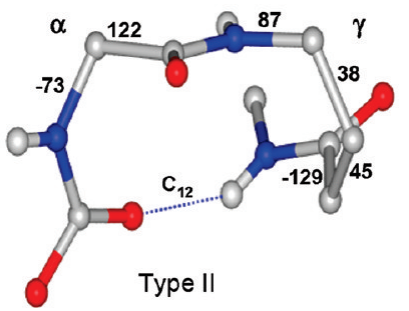

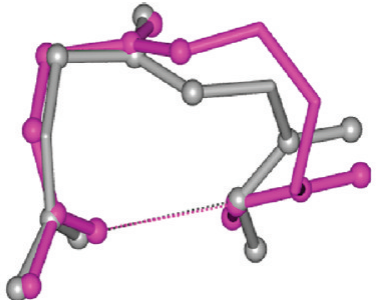

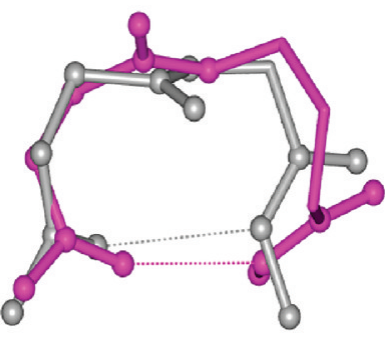

FIGURE 8. $\alpha \gamma \mathrm{C}_{12}$ hydrogen-bonded turns and their superposition with the type I and type II $\beta$-turns formed in $\alpha \alpha$ segments: (a) type I (helical) $\mathrm{C}_{12}$ turn (left) and its (magenta) superposition with a type I $\beta$-turn $\left(\phi_{i+1}=-55^{\circ}, \psi_{i+1}=-39^{\circ} ; \phi_{i+2}=-83^{\circ}, \psi_{i+2}=4.5^{\circ}\right.$, gray $)$ formed in $\alpha \alpha$ segments (right); (b) type II $\alpha \gamma \mathrm{C}_{12}$ turn (left) and its (magenta) superposition with a type II $\beta$-turn $\left(\phi_{i+1}=-59.6^{\circ}, \psi_{i+1}=\right.$ $126.8^{\circ} ; \phi_{i+2}=92.9^{\circ}, \psi_{i+2}=-25.8^{\circ}$; gray) formed in $\alpha \alpha$ segments (right). The atoms used for the superposition are shown as balls.

larger number of intramolecular hydrogen bonds are favored in poorly solvating media like chloroform $\left(\mathrm{CDCl}_{3}\right)$, $\beta$-hairpins with fewer internal hydrogen bonds are favored in solvents that can interact with exposed hydrogen bond donors and acceptors on the peptide backbone. In proteins, $\beta$-hairpins in which the antiparallel strands are connected by two and three residue loops (turns) are widespread. ${ }^{31}$ Two residue $(\alpha \alpha)$ segments are generally stabilized by $C_{10}$ hydrogen bonds, while $C_{13}$ hydrogen bonds occur in three residue $(\alpha \alpha \alpha)$ segments. ${ }^{37}$ Connecting loops in $\beta$-hairpins can be readily constructed using hybrid peptide segments. The $\alpha \gamma$ segment in the peptide hairpin shown in Figure 9c is an example of a $C_{12}$ loop, while a central $C_{13}(\alpha \delta)$ turn has been established in crystals for the PPro- $\delta$ Ava $(\delta$ Ava, $\delta$-aminovaleric acid) segment in the octapeptide Boc-LeuVal-Val-DPro- $\delta$ Ava-Leu-Val-Val-OMe. ${ }^{38}$

Crystal structure determinations of short Gpn peptides reveal larger hydrogen-bonded ring sizes. $\mathrm{A}_{13}$ intramolecular hydrogen bond is observed in crystals of Boc- $\beta$ Leu-GpnVal-OMe and Boc- $\beta$ Phe-Gpn-Phe-OMe (Figure 10). ${ }^{27}$ These two $\mathrm{C}_{13}$ hydrogen-bonded $\beta \gamma$ turns correspond to two distinct conformational classes. The structural feature observed for the $\beta$ Leu-Gpn segment can be repeated to generate a continuous $\mathrm{C}_{13}$ hydrogen-bonded helix, corresponding to a hybrid analog of the canonical $\alpha$-helix, in which the three-residue $\alpha \alpha \alpha$ repeat has been replaced by a two-residue hybrid $\beta \gamma$ seg- 


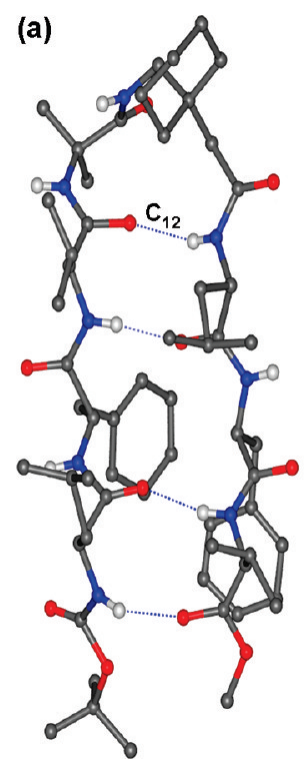

(b)

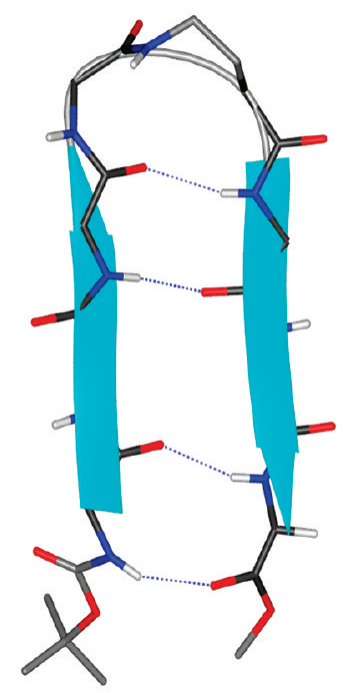

(c)

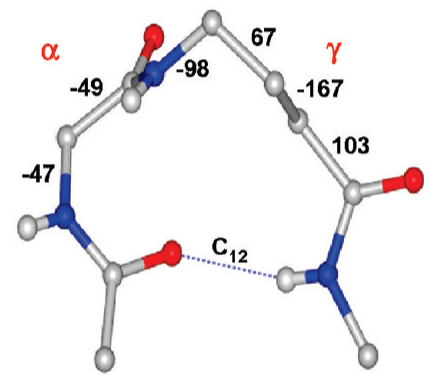

FIGURE 9. (a) The $\beta$-hairpin structure determined in the crystals of Boc-Leu-Phe-Val-Aib-Gpn-Leu-Phe-Val-OMe, ${ }^{32}$ (b) ribbon representation of the $\beta$-hairpin, and (c) the central $\alpha \gamma \mathrm{C}_{12}$ hydrogen-bonded turn, with backbone torsion angles marked.

(a)

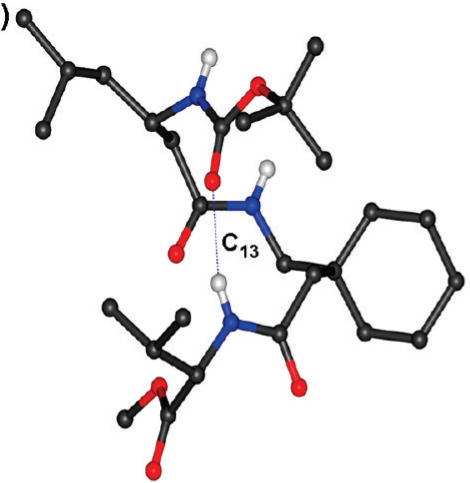

(c)

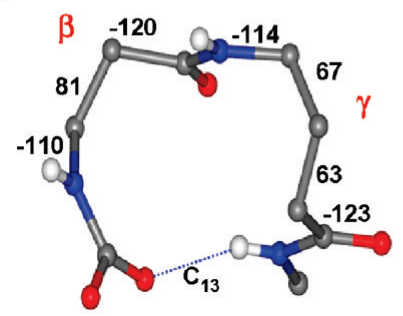

(b)

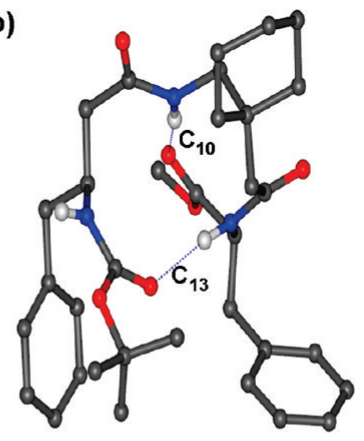

(d)

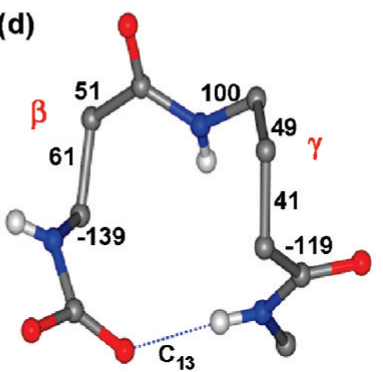

(e)

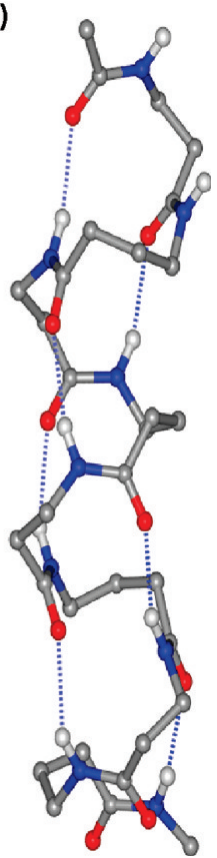

FIGURE 10. $\mathrm{C}_{13}$ hydrogen-bonded structures characterized in $\beta \gamma$ hybrid segments of peptides containing Gpn: (a) Boc- $\beta$ Leu-Gpn-Val-OMe, ${ }^{27}$ helical $\mathrm{C}_{13}$ turn, (b) Boc- $\beta$ Phe-Gpn-Phe-OMe, ${ }^{27}$ nonhelical $\mathrm{C}_{13}$ turn, (c) Boc- $\beta$ Leu-Gpn-Val-OMe $(\beta \gamma) \mathrm{C}_{13}$ turn, and (d) Boc- $\beta$ Phe-Gpn-Phe-OMe $(\beta \gamma) \mathrm{C}_{13}$ turn. Backbone torsion angles (deg) are marked. (e) $\beta \gamma \mathrm{C}_{13}$ helix built using the parameters of the $\mathrm{C}_{13}$ hydrogen bond shown in panel $\mathrm{C}$.

ment (Figure 10c). It should be noted that the number of backbone atoms in $\alpha \alpha \alpha$ and $\beta \gamma$ segments are identical. The unsubstituted $\beta \gamma$ segment $\beta$ Ala $-\gamma$ Abu also adopts the $\mathrm{C}_{13}$ helical conformation when embedded into a host $\alpha$-peptide helix. ${ }^{39}$ An alternative "nonhelical" $\beta \gamma \mathrm{C}_{13}$ hydrogen-bonded turn is observed in crystals of the $\beta \gamma \alpha$ tripeptide Boc- $\beta$ PheGpn-Phe-OMe. ${ }^{27}$ This hybrid unit containing three different $\omega$ amino acid residues adopts an interesting two hydrogen- bonded structure containing the $\mathrm{C}_{13} \beta \gamma$ turn followed by a $\mathrm{C}_{10}$ $\gamma \alpha$ turn with reversed hydrogen bond directionality. This type of $\mathrm{C}_{10}$ hydrogen bonding in a $\gamma \alpha$ segment has also been characterized in $\alpha \gamma \alpha$ sequences (Figure 7). ${ }^{34}$

A very large $C_{17}$ hydrogen-bonded turn has been observed for a $\gamma \alpha \gamma$ segment in the peptide Boc-Aib-Gpn-Aib-Gpn-NHMe (Figure 11). ${ }^{40}$ This structural feature places $C^{\alpha}{ }_{(i)}$ and $C^{\alpha}{ }_{(i+4)}$ at a distance of $5.2 \AA$, which is very close to that observed at the 
(a)

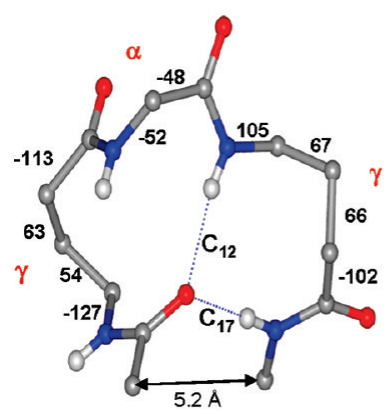

(b)

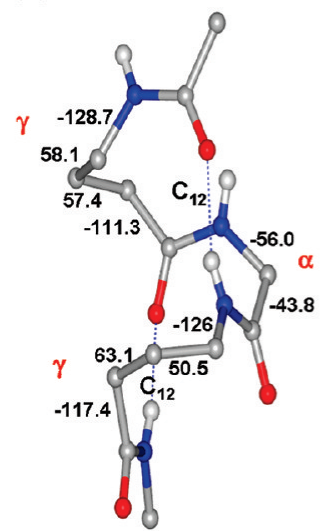

(c)

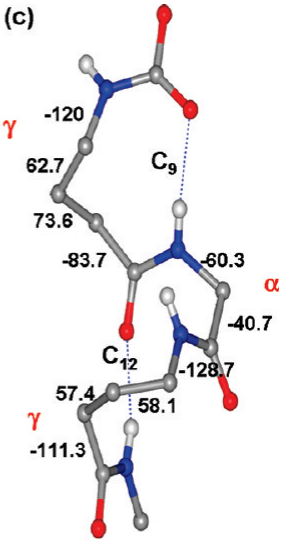

(d)

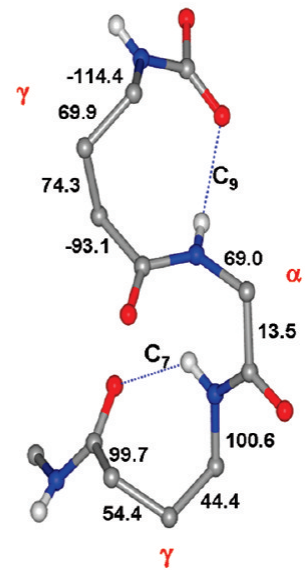

(e)

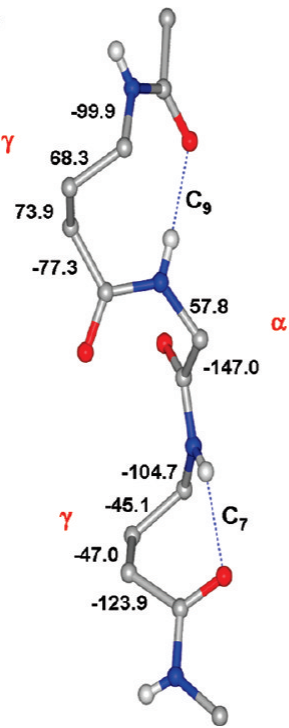

FIGURE 11. Hydrogen-bonded structures observed in crystals for the $\gamma \alpha \gamma$ segment: (a) $\gamma \alpha \gamma \mathrm{C}_{17}$ hydrogen-bonded turn in Boc-Aib-Gpn-AibGpn-NHMe, polymorph $A_{;}^{40}$ (b) $C_{12}$ helical conformation and (c) $C_{9} / C_{12}$ conformation of the $\gamma \alpha \gamma$ segments from the helix, Boc-Gpn-Aib-GpnAib-Gpn-Aib-Gpn-Aib-OMe, ${ }^{32}$ (d) the $\mathrm{C}_{9} / \mathrm{C}_{7}$ hydrogen-bonded conformation in Boc-Gpn-Aib-Gpn-Aib-OMe ${ }^{27}$ and (e) in the polymorph B/C of Boc-Aib-Gpn-Aib-Gpn-NHMe. ${ }^{40}$ Backbone torsion angles (deg) are marked.

turn segment in an antiparallel $\beta$-hairpin. Thus, the 17 atom hydrogen-bonded turn could, in principle, mimic the larger connecting loops observed for $\beta$-hairpins in protein structures. For example, a four residue $\alpha \alpha \alpha \alpha$ connecting loop would contain 16 atoms in the hydrogen-bonded loop. Interestingly, several conformational states that contain two intramolecular hydrogen bonds have been characterized in crystals for the segment -Gpn-Aib-Gpn-. The steric constraints present at both the $\alpha$ and $\gamma$ amino acid residues precludes the formation of extended strand conformations, which are held together by $\beta$-sheet hydrogen-bonding patterns, commonly observed in short $\alpha$ peptide sequences that contain the corresponding $\alpha \alpha \alpha$ segment. As illustrated in Figure 11, as many as five distinct conformational states have been captured in crystals for the $\gamma \alpha \gamma$ segment. Two dramatically different states are the folded $\mathrm{C}_{12} / \mathrm{C}_{17}$ hydrogen-bonded structure, ${ }^{40}$ which permits complete reversal of the polypeptide chain direction (Figure 11a), and the $C_{12} / C_{12}$ hydrogen-bonded structure $\left(C_{12}\right.$ helix, Figure $11 b){ }^{32}$ The potential for the transition between a hairpin loop facilitating a $180^{\circ}$ chain reversal and a continuous helical structure effectively requires a major change only in the Gpn(3) torsion angle $\phi$. The $C_{9} / C_{12}$ conformation (Figure 11c) is very closely related to the $C_{12}$ helix, with small changes in the torsion angles resulting in a change in the hydrogen-bonding pattern. ${ }^{32}$ Conformational transitions that require movement of a residue only within a limited region of conformational space are likely to be kinetically more accessible than large-scale movements, which may require traversing of appreciable activation barriers. The $C_{9} / C_{7}$ structures shown in Figure $11 \mathrm{~d}$,e are partially unraveled structures, ${ }^{40}$ in which the flanking Gpn residues form intramolecular hydrogen bonds, determined only by local residue conformations with the central $\alpha$ residue serving only as a linker. These well-characterized conformational states illustrate the multiplicity of 

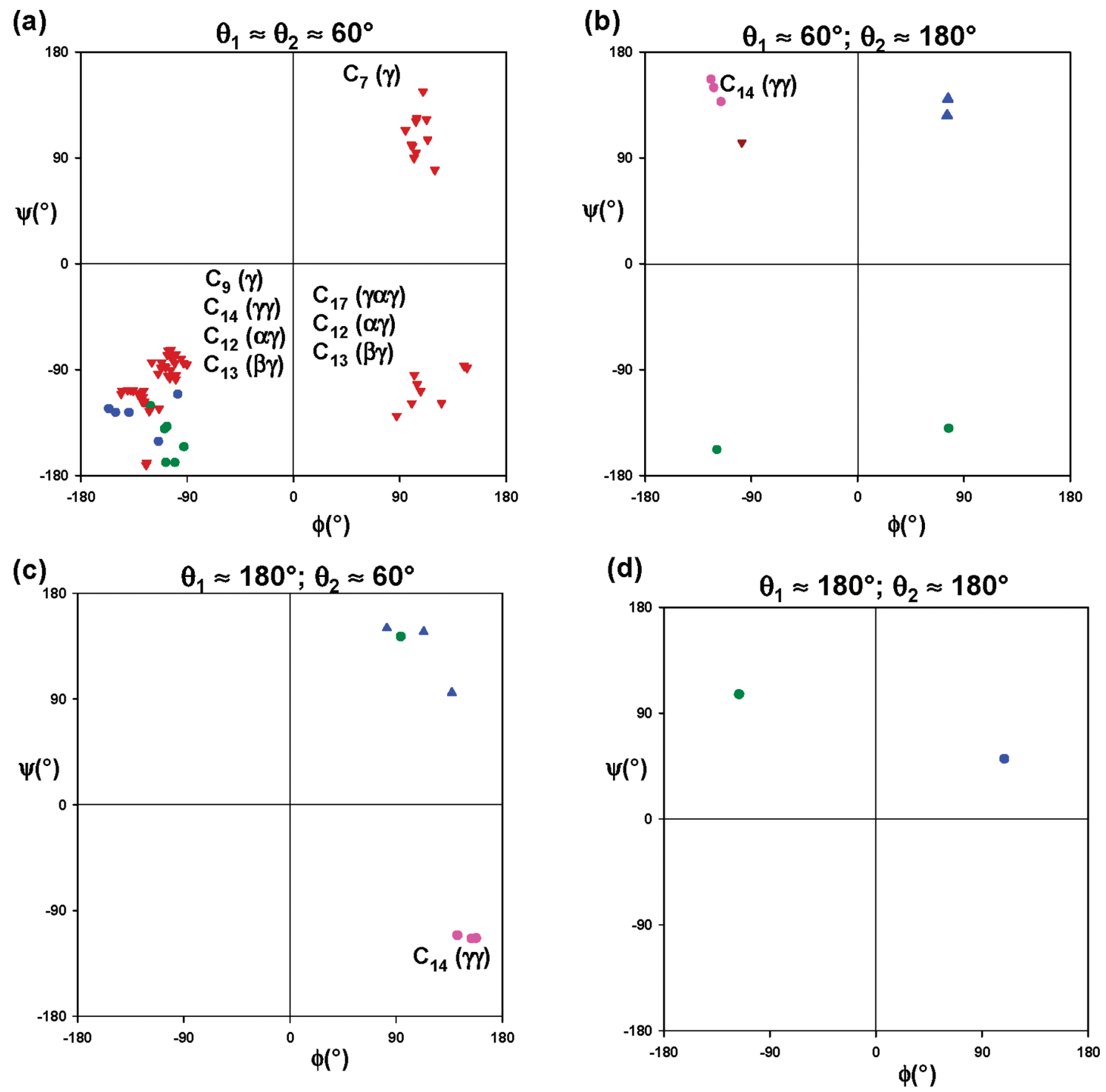

\section{$\gamma$ Abu $\gamma^{2,3,4}$-trisubstituted residues \\ trans-3-ACPC residue $\quad \nabla$ Gpn}

FIGURE 12. The $\phi-\psi$ scatter plots for crystallographically characterized $\gamma$-residues, for various $\theta_{1}$ and $\theta_{2}$ combinations. Intramolecular hydrogen bonds involving the $\gamma$-residues are indicated.

internally hydrogen-bonded structures that may be generated in hybrid sequences containing $\alpha$ and $\gamma$ amino acid residues.

Figure 12 summarizes the available conformational distribution observed in crystal structures for a range of $\gamma$ residues. Two dimensional $\phi-\psi$ scatter plots for fixed combinations of the dihedral angles $\theta_{1}$ and $\theta_{2}$ permit inspection of clustering in specific regions of conformational space. Thus far, the largest body of experimental data is for Gpn residues, with the gauche-gauche conformation being the most represented. From Figure 12a, it is evident that all helical/ribbon structures that incorporate conventional hydrogen bond direction $\left(\mathrm{CO}_{i} \cdots \mathrm{NH}_{i+n}, n=1,2,3\right.$ etc.) cluster into the lower left quad- 
rant of $\phi-\psi$ space for the $g^{+} g^{+}$combination for $\gamma$ residues. The cluster obtained in the bottom right quadrant represents the $\gamma$ residue conformations in internally hydrogen-bonded hybrid segments, which can be accommodated in loops, resulting in polypeptide chain reversals as well as in helices with mixed hydrogen bond directionalities like the $C_{12} / C_{10}$ helix in $(\alpha \gamma)_{n}$ sequences. ${ }^{34}$ The $C_{7}$ ribbon in which the $\gamma$ residue occupies the top right quadrant requires a distinct backbone conformation. It should be noted that for an $\alpha \gamma$ segment, two distinct $C_{12}$ hydrogen-bonded conformations have been characterized in crystal structures of Gpn peptides. Repetition of the more abundantly observed type I conformation ( $\alpha$ residue, $\phi=-63^{\circ}, \psi=-32^{\circ}$; $\gamma$ residue, $\phi=-132^{\circ}, \theta_{1}=54^{\circ}$, $\theta_{2}=60^{\circ}, \psi=-112^{\circ}$ ) leads to the $C_{12} \alpha \gamma$ helix, which is an expanded analog of the $\alpha$ polypeptide $3_{10}$-helix. ${ }^{32}$ The type II $\alpha \gamma$ turn ( $\alpha$ residue, $\phi=-72^{\circ}, \psi=121^{\circ} ; \gamma$ residue, $\phi=87^{\circ}$, $\theta_{1}=38^{\circ}, \theta_{2}=45^{\circ}, \psi=-129^{\circ}$ ) can also be repeated to yield the $C_{12} / C_{10}$ helix with mixed hydrogen bond directionalities. ${ }^{34}$ There is no analog of the $C_{12} / C_{10}$ structure in the all $\alpha$ peptide sequences because the $\mathrm{C}_{8}$ conformation at the $\alpha \alpha$ segment is strained and energetically unfavorable. ${ }^{41}$ However, in all $\beta$-peptides containing alternate $\beta^{3}$ and $\beta^{2}$ residues, solution NMR studies support the formation of a mixed 12/10helix analogous to the $\alpha \gamma$ structure described above. ${ }^{42,43}$ The experimentally determined backbone torsion angles for the $\alpha \gamma$ $\mathrm{C}_{12}$ helix, the $\alpha \gamma \mathrm{C}_{12} / \mathrm{C}_{10}$ mixed helix and the $\beta \gamma \mathrm{C}_{13}$ helix are in good agreement with theoretical estimates from calculations. ${ }^{44}$ Figure 12 also shows the distribution of $\gamma$ residue $\phi$, $\psi$ values for the less frequently observed combinations of $\theta_{1}$ and $\theta_{2}$, namely, $g t, t g$, and $t t$. For the simple two residue $\gamma \gamma$ segment, there are undoubtedly several possibilities for internal hydrogen bond formation. The work of Seebach and coworkers using multiply substituted $\gamma$ amino acid residues provides two interesting examples. In a $\gamma$ tetrapeptide crystal structure, an incipient $C_{14}$ helix has been characterized with two successive $C_{14}$ hydrogen bonds, ${ }^{45}$ a conformation that constitutes an expansion of the analogous $3_{10}$-helix in $\alpha$ polypeptides. The crystal structure of a $\gamma$ dipeptide provides an example of a $\mathrm{C}_{14}$ hydrogen bond in which the turn residues adopt $g t$ and $t g$ conformations. ${ }^{46}$

\section{Conformational Diversity in Gpn Peptides}

Despite the steric constraints imposed by the geminal dialkyl substituents at $C^{\beta}$, Gpn residues can sample significantly diverse regions of conformational space. The behavior of Gpn may be contrasted with that of the $\alpha$ amino acid residue Aib, which carries gem dialkyl substituents at $C^{\alpha}$. In the case of Aib, the $\alpha_{L}$ and $\alpha_{R}$ regions of $\phi-\psi$ space are overwhelmingly pop- (a)

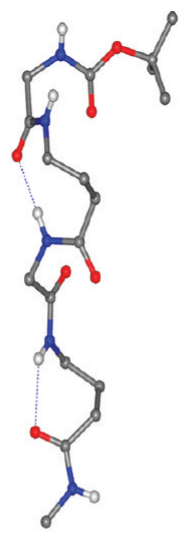

(c)
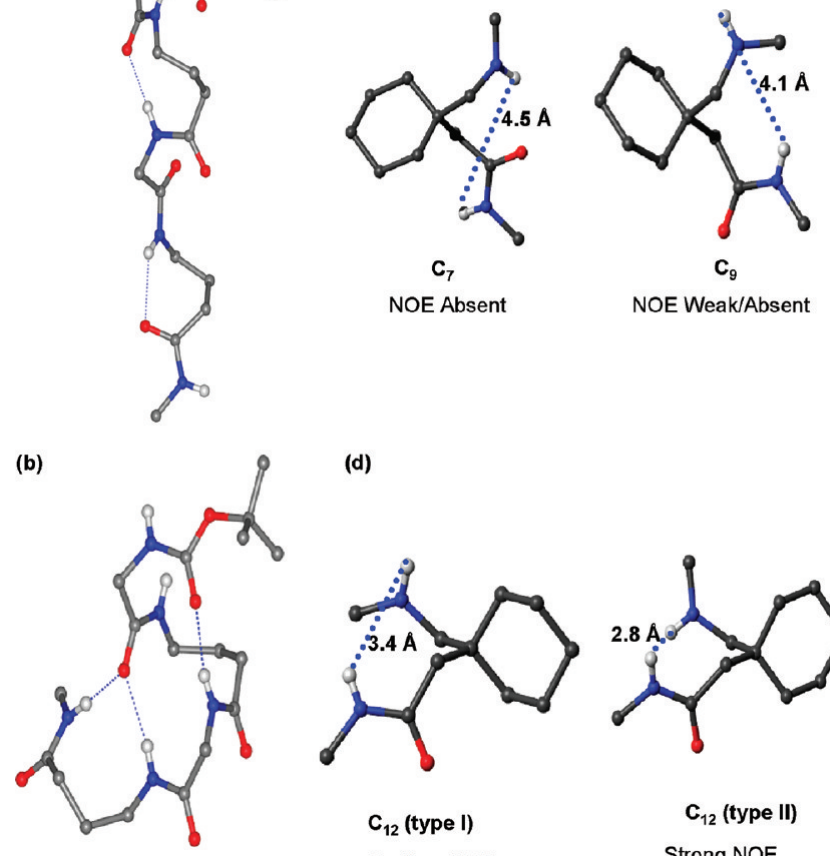

(d)
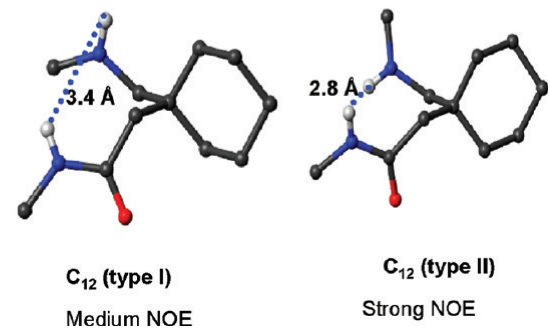

FIGURE 13. (a, b) Two distinct molecular conformations observed for Boc-Aib-Gpn-Aib-Gpn-NHMe in three polymorphic crystals. ${ }^{40}$ Only the backbone atoms are shown, for clarity. The conformation in panel a is stabilized by a $C_{9}$ and a $C_{7}$ hydrogen bond, formed at $\mathrm{Gpn}(2)$ and $\mathrm{Gpn}(4)$ residues, respectively. In the conformation in panel $b$, the Gpn(2) residue is involved in a type I $\alpha \gamma \mathrm{C}_{12}$ (helical) hydrogen bond and a three-residue, $\gamma \alpha \gamma \mathrm{C}_{17}$ hydrogen bond, while the $\mathrm{Gpn}(4)$ residue is involved only in the $\mathrm{C}_{17}$ hydrogen bond. The backbone conformation of the Gpn(4) residue is close to that of the type II $\alpha \gamma \mathrm{C}_{12}$ turn. (c, d) The $\mathrm{NH}_{i}-\mathrm{NH}_{i+1}\left(d_{\mathrm{NN}}\right)$ distances across the $\gamma$ residues with different backbone conformations: (c) $d_{\mathrm{NN}}$ in $\mathrm{C}_{7}$ (left) and $\mathrm{C}_{9}$ (right) hydrogen-bonded conformations; (d) $d_{\mathrm{NN}}$ in $\alpha \gamma$ hybrid $\mathrm{C}_{12}$ turns, type I (helical, left) and type II (in $\mathrm{C}_{12} / \mathrm{C}_{10}$ mixed helix, right).

ulated. The polyproline $\left(\mathrm{P}_{\mathrm{II}}\right)$ and fully extended conformations are seen very infrequently in very short peptides. ${ }^{47}$ The presence of variable torsion angles in Gpn residues significantly enhances the possibility of obtaining alternative conformations that facilitate intramolecular hydrogen bond formation in both homo-oligomeric and hybrid peptide sequences. Conformational diversity manifests itself in the solid state when polymorphic crystals are obtained, in which peptides reveal entirely different backbone conformations. This feature is illustrated by the peptide Boc-Aib-Gpn-Aib-Gpn-NHMe, which crystallizes in three distinct polymorphic forms. ${ }^{40}$ Two distinct conformational states have been characterized as shown in Figure 13. The two Gpn residues in these two distinct structures provide examples of $C_{7}, C_{9}, C_{12}(\alpha \gamma)$, and $C_{17}(\gamma \alpha \gamma)$ internally hydrogen-bonded conformations. In the solution state, exchange between multiple conformations can influence the 
observed NMR parameters, which are used to establish local residue conformations. In the case of Gpn residues, the transition from one region of $\phi-\psi$ space to another for a fixed $g g$ combination of $\theta_{1}$ and $\theta_{2}$ can involve appreciable activation barriers. As a consequence, the intermediate and slow exchange regimes are quite frequently observed in solution NMR spectra for peptides containing Gpn residues, resulting in the observation of selective line broadening particularly for backbone amide protons and the detection of minor resonances. When conformational exchange is rapid compared with the NMR time scale, dynamic averaging effects can also influence observed nuclear Overhauser effects (NOEs). ${ }^{33}$ As shown in Figure 13, the sequential $\mathrm{NH}_{i}-\mathrm{NH}_{i+1}\left(d_{\mathrm{NN}}\right)$ distance across the Gpn residue can vary significantly, depending on the values of the backbone torsion angles. Solution NMR studies for both homo-oligomeric and hybrid sequences containing $\gamma$ residues must be interpreted with caution since conformational heterogeneity may play a role. We restricted our studies so far to nonaqueous solvents, where the driving forces for folding are predominantly inter-residue hydrogen bond formation and nonbonded interactions, determining the local conformations of individual residues. Extension of these studies to water-soluble peptides must necessarily take into account the role of hydrophobic effects in influencing backbone folding.

Gabapentin (Gpn) is a readily available and versatile $\gamma$ amino acid, which can be used in the design of well-folded hybrid peptides. The absence of chirality and the constraints imposed on the torsion angles $\theta_{1}$ and $\theta_{2}$ by symmetrical substitution at the $C^{\beta}$ atom largely restrict the Gpn residue to adopting gauche-gauche conformations about the two additional $\mathrm{C}-\mathrm{C}$ bonds that have been introduced into the polypeptide backbone. A variety of internally hydrogen-bonded structures have been demonstrated in the solid state for Gpncontaining peptides. These include the characterization of the $C_{7}$ and $C_{9}$ hydrogen bonds, which can lead to ribbons in homo-oligomeric sequences. In hybrid $\alpha \gamma$ sequences, distinct $\mathrm{C}_{12}$ hydrogen-bonded turn structures have been established that support formation of peptide helices and hairpins in longer sequences. The use of the Gpn residue in $(\alpha \gamma)_{n}$ sequences has permitted the experimental characterization of the $\alpha \gamma \mathrm{C}_{12}$ helix in which hydrogen bond directionality is the same as that in $\alpha$ peptide helices and also the $C_{12} / C_{10}$ helix in which the directionality of the 12 -atom and 10 -atom hydrogen bonds are opposite. The diversity of internally hydrogenbonded structures observed in hybrid sequences containing
Gpn augurs well for the use of this residue in the rational design of novel peptide structures incorporating hybrid backbones.

We thank Dr. K. Nagarajan, Hikal RaD Centre, Bangalore, for introducing us to gabapentin and for gifts of the amino acid. This research was supported by a grant from the Council of Scientific and Industrial Research (CSIR) and Program Support in the area of Molecular Diversity and Design, Department of Biotechnology, India. P.G.V. and S.C. thank the CSIR for Senior Research Fellowships.

\section{BIOGRAPHICAL INFORMATION}

Prema G. Vasudev obtained a M.Sc. degree in Organic Chemistry from Mahatma Gandhi University in 2001. She is a research student in the Department of Physics, Indian Institute of Science.

Sunanda Chatterjee obtained a M.Sc. degree in Organic Chemistry from University of Burdwan in 2003. She is a research student in the Molecular Biophysics Unit, Indian Institute of Science.

Narayanaswamy Shamala has a M.Sc. degree from the University of Mysore and Ph.D. from University of Madras, India. Subsequently, she joined the faculty of the Indian Institute of Science, where she is presently a Professor in the Department of Physics.

Padmanabhan Balaram has a M.Sc. degree from the IIT, Kanpur, and Ph. D from Carnegie-Mellon University. Subsequently, he joined the faculty of the Indian Institute of Science, where he is presently a Professor of Molecular Biophysics.

\section{FOOTNOTES}

*To whom correspondence should be addressed. E-mail addresses: shamala@ physics.iisc.ernet.in; pb@mbu.iisc.ernet.in.

\section{REFERENCES}

1 Seebach, D.; Matthews, J. L. $\beta$-Peptides: A Surprise at Every Turn. Chem. Commun. 1997, 2015-2022.

2 Gellman, S. H. Foldamers: A Manifesto. Acc. Chem. Res. 1998, 31, 173-180.

3 Cheng, R. P.; Gellman, S. H.; DeGrado, W. F. $\beta$-Peptides: From Structure to Function. Chem. Rev. 2001, 101, 3219-3232.

4 Seebach, D.; Beck, A. K.; Bierbaum, D. J. The World of $\beta$ - and $\gamma$-Peptides Comprised of Homologated Proteinogenic Amino Acids and other Components. Chem. Biodivers. 2004, 1, 1111-1239.

5 Roy, R. S.; Balaram, P. Conformational Properties of Hybrid Peptides Containing $\alpha$ and $\omega$-Amino Acids. J. Pept. Res. 2004, 63, 279-289.

6 Seebach, D.; Hook, D. F.; Glättli, A. Helices and Other Secondary Structures of $\beta$ and $\gamma$-Peptides. Biopolymers (Pept. Sci.) 2006, 84, 23-37.

7 Horne, W. S.; Gellman, S. H. Foldamers with Heterogenous Backbones. Acc. Chem. Res. 2008, 41, 1399-1408.

8 Chatterjee, S.; Roy, R. S.; Balaram, P. Expanding the Polypeptide Backbone: Hydrogen Bonded Conformations in Hybrid Polypeptides Containing the Higher Homologues of $\alpha$-Amino Acids. J. R. Soc. Interface 2007, 4, 587-606.

9 Goodman, C. M.; Choi, S.; Shandler, S.; DeGrado, W. F. Foldamers as Versatile Frameworks for the Design and Evolution of Function. Nat. Chem. Biol. 2007, 3, 252-262.

10 Banerjee, A.; Balaram, P. Stereochemistry of Peptides and Polypeptides Containing $\omega$-Amino Acids. Curr. Sci. 1997, 73, 1067-1077.

11 Seebach, D.; Gardiner, J. Peptidic Peptidomimetics. Acc. Chem. Res. 2008, 41, 1366-1375.

12 Stephens, 0.; Kim, S.; Welch, B. D.; Hodsdon, M. E.; Kay, M. S.; Schepartz, A. Inhibiting HIV Fusion with a $\beta$-Peptide Foldamer. J. Am. Chem. Soc. 2005, 127, 13126-13127. 
13 Horne, W. S.; Boersma, M. D.; Windsor, M. A.; Gellman, S. H. Sequence-Based Design of $\alpha / \beta$-Peptide Foldamers that Mimic BH3 Domains. Angew. Chem., Int. Ed. 2008, 47, 2853-2856.

14 Venkatraman, J.; Shankaramma, S. C.; Balaram, P. Design of Folded Peptides. Chem. Rev. 2001, 101, 3131-3152.

15 Prasad, B. V. V.; Balaram, P. The Stereochemistry of $\alpha$-Aminoisobutyric Acid Containing Peptides. CRC Crit. Rev. Biochem. 1984, 16, 307-347.

16 Karle, I. L.; Balaram, P. Structural Characteristics of $\alpha$-Helical Peptide Molecules Containing Aib Residues. Biochemistry 1990, 29, 6747-6756.

17 Toniolo, C.; Benedetti, E. The Polypeptide 310-Helix. Trends Biochem. Sci. 1991, 16, 350-353.

18 Aravinda, S.; Shamala, N.; Roy, R. S.; Balaram, P. Non-Protein Amino Acids in Peptide Design. Proc. Indian Acad. Sci. (Chem. Sci.) 2003, 115, 373-400.

19 Sills, G. J. The Mechanism of Action of Gabapentin and Pregabalin. Curr. Opin. Pharmacol. 2006, 6, 108-113.

20 Milner-White, E. J. Situations of $\gamma$-Turns in Proteins: Their Relation to $\alpha$-Helices, $\beta$-Sheets and Ligand Binding Sites. J. Mol. Biol. 1990, 216, 385-397.

21 Toniolo, C.; Benedetti, E. Fully Extended Polypeptide Conformation. In Molecular Conformation and Biological Interactions, Balaram, P., Ramaseshan, S., Eds.; Indian Academy of Sciences: Bangalore: 1991, pp 511-521.

22 Jimenez, A. I.; Ballano, G.; Cativiela, C. First Observation of Two Consecutive $\gamma$ Turns in a Crystalline Linear Dipeptide. Angew. Chem., Int. Ed. 2005, 44, 396-399.

23 Vasudev, P. G.; Rai, R.; Shamala, N.; Balaram, P. Conformations of $\beta$-Amino Acid Residues in Peptides: $X$-ray Diffraction Studies of Peptides Containing the Achiral Residue 1-Aminocyclohexaneacetic Acid, $\beta^{3,3}{ }^{3} c_{6}$ C. Biopolymers (Pept. Sci.) 2008, 90, 138-150

24 Seebach, D.; Abele, S.; Sifferlen, T.; Hanggi, M.; Gruner, S.; Seiler, P. Preparation and Structure of $\beta$-Peptides Consisting of Geminally Disubstituted $\beta^{2,2_{2}}$ and $\beta^{3,3}$ Amino Acids: A Turn Motif for $\beta$-Peptides. Helv. Chim. Acta 1998, 81, 2218-2243.

25 Vasudev, P. G.; Ananda, K.; Shamala, N.; Balaram, P. C. Helices and Ribbons in $\gamma$ Peptides. Angew. Chem., Int. Ed. 2005, 44, 4972-4975.

26 Ananda, K.; Aravinda, S.; Vasudev, P. G.; Raja, K. M. P.; Sivaramakrishnan, H.; Nagarajan, K.; Shamala, N.; Balaram, P. Stereochemistry of Gabapentin and Several Derivatives. Solid State Conformations and Solution Equilibria. Curr. Sci. 2003, 85, 1002-1011.

27 Vasudev, P. G.; Ananda, K.; Chatterjee, S.; Aravinda, S.; Shamala, N.; Balaram, P. Hybrid Peptide Design. Hydrogen Bonded Conformations in Peptides Containing the Stereochemically Constrained $\gamma$-Amino Acid Residue, Gabapentin. J. Am. Chem. Soc. 2007, 129, 4039-4048.

28 Ananda, K.; Vasudev, P. G.; Sengupta, A.; Raja, K. M. P.; Shamala, N.; Balaram, P. Polypeptide Helices in Hybrid Peptide Sequences. J. Am. Chem. Soc. 2005, 127, 16668-16674.

29 Venkatachalam, C. M. Stereochemical Criteria for Polypeptides and Proteins. V. Conformation of a System of Three Linked Peptide Units. Biopolymers 1968, 6, 1425-1436.

30 Donohue, J. Hydrogen Bonded Helical Configurations of the Polypeptide Chain. Proc. Natt. Acad. Sci. U.S.A. 1953, 39, 470-478.

31 Gunasekaran, K.; Ramakrishnan, C.; Balaram, P. $\beta$-Hairpins in Proteins Revisited: Lessons for de Novo Design. Protein Eng. 1997, 10, 1131-1141.

32 Chatteriee, S.; Vasudev, P. G.: Raghothama, S.; Ramakrishnan, C.: Shamala, N.; Balaram, P. Expanding the Peptide $\beta$-Turn in $\alpha \gamma$ Hybrid Sequences: 12 Atom Hydrogen Bonded Helical and Hairpin Turns. J. Am. Chem. Soc. 2009, 131, 59565965.
33 Chatterjee, S.; Vasudev, P. G.; Raghothama, S.; Shamala, N.; Balaram, P. Solid State and Solution Conformations of a Hybrid $\alpha \gamma \alpha \alpha \gamma \alpha$ Hexapeptide. Characterization of a Backbone Expanded Analog of the $\alpha$-Polypeptide $3{ }_{10}$ - Helix. Biopolymers (Pept. Sci.) 2008, 90, 759-771.

34 Vasudev, P. G.; Chatterjee, S.; Ananda, K.; Shamala, N.; Balaram, P. Hybrid $\alpha \gamma$ Polypeptides: Structural Characterization of a $\mathrm{C}_{12} / \mathrm{C}_{10}$ Helix with Alternating Hydrogen Bond Polarity. Angew. Chem., Int. Ed. 2008, 47, 6430-6432.

35 Gunasekaran, K.; Gomathi, L.; Ramakrishnan, C.; Chandrasekhar, J.; Balaram, P. Conformational Interconversions in Peptide $\beta$-Turns: Analysis of Turns in Proteins and Computational Estimates of Barriers. J. Mol. Biol. 1998, 284, 1505-1516.

36 Rai, R.; Raghothama, S.; Sridharan, R.; Balaram, P. Tuning the $\beta$-Turn Segment in Designed Peptide $\beta$-Hairpins: Construction of a Stable Type l' $\beta$-Turn Nucleus and Hairpin-Helix Transition Promoting Segments. Biopolymers 2007, $88,350-361$

37 Rai, R.; Raghothama, S.; Balaram, P. Design of a Peptide Hairpin Containing a Central Three Residue Loop. J. Am. Chem. Soc. 2006, 128, 2675-2681.

38 Rai, R.; Vasudev, P. G.; Ananda, K.; Raghothama, S.; Shamala, N.; Karle, I. L.; Balaram, P. Hybrid Peptides: Expanding the $\beta$-Turn in Peptide Hairpins by Insertion of $\beta, \gamma$ and $\delta$ Residues. Chem.-Eur. J. 2007, 13, 5917-5926.

39 Karle, I. L.; Pramanik, A.; Banerjee, A.; Battacharjya, S.; Balaram, P. $\omega$-Amino Acids in Peptide Design. Crystal Structures and Solution Conformations of Peptide Helices Containing a $\beta$-Alanyl- $\gamma$-aminobutyryl Segment. J. Am. Chem. Soc. 1997, 119, 9087-9095.

40 Chatterjee, S.; Vasudev, P. G.; Ananda, K.; Raghothama, S.; Shamala, N.; Balaram, P. Multiple Conformational States in Crystals and in Solution in $\alpha \gamma$ Hybrid Peptides. Fragility of the $\mathrm{C}_{12}$ Helix in Short Sequences. J. Org. Chem. 2008, 73, 6595-6606.

41 Rao, P.; Nagaraj, R.; Rao, C. N. R.; Balaram, P. Infrared Spectroscopy as a Probe for the Development of Secondary Structure in the Amino Terminal Segment of Alamethicin. FEBS Lett. 1979, 100, 244-248.

42 Seebach, D.; Gademann, K.; Schreiber, J. V.; Matthews, J. L.; Hintermann, T.; Jaun, B.; Oberer, L.; Hommel, U.; Widmer, H. Mixed $\beta$-Peptides: A Unique Helical Secondary Structure in Solution. Helv. Chim. Acta 1997, 80, 2033-2038.

43 Seebach, D.; Abele, S.; Gademann, K.; Guichard, G.; Hintermann, T.; Jaun, B.; Matthews, J. L.; Schreiber, J. V.; Oberer, L.; Hommel, U.; Widmer, H. $\beta^{2}$ - and $\beta^{3}$ Peptides with Proteinaceous Side Chains: Synthesis and Solution Structures of Constitutional Isomers, a Novel Helical Secondary Structure and the Influence of Solvation and Hydrophobic Interactions on Folding. Helv. Chim. Acta 1998, 81, 932-982.

44 Baldauf, C.; Gunther, R.; Hofmann, H.-J. Helix Formation in $\alpha, \gamma$ - and $\beta, \gamma$-Hybrid Peptides: Theoretical Insights into Mimicry of $\alpha$ - and $\beta$-Peptides. J. Org. Chem. 2006, 71, 1200-1208.

45 Seebach, D.; Brenner, M.; Rueping, M.; Jaun, B. $\gamma^{2}-, \gamma^{3}$, and $\gamma^{2,3,4}$-Amino Acids, Coupling to $\gamma$-Hexapeptides: CD Spectra, NMR Solution and X-ray Crystal Structures of $\gamma$-Peptides. Chem.-Eur. J. 2002, 8, 573-584.

46 Brenner, M.; Seebach, D. Design, Synthesis, NMR-Solution and X-ray Crystal Structure of $N$-Acyl- $\gamma$-dipeptide Amides That Form a $\beta \| l^{\prime}$-Type Turn. Helv. Chim. Acta 2001, 84, 2155-2166.

47 Aravinda, S.; Shamala, N.; Balaram, P. Aib Residues in Peptaibiotics and Synthetic Sequences. Analysis of Nonhelical Conformations. Chem. Biodivers. 2008, 5, 1238-1262. 